\title{
MULTISPECTRAL REMOTE SENSING IMAGE CLASSIFICATION BASED ON QUANTUM ENTANGLEMENT
}

\author{
Fan Yang ${ }^{1,2,3}$, Guoqing Zhou ${ }^{1,2}$, Jianrong Xiao ${ }^{1,3^{*}}$, Qi $\mathrm{Li}^{1,2,3}$, Bin $\mathrm{Jia}^{1,2}$, Haoyu Wang ${ }^{1,2}$, Jian Gao ${ }^{1,2}$ \\ ${ }^{1}$ Guangxi Key Laboratory of Spatial Information and Geomatics, Guilin University of Technology, No. 12 Jian'gan Road, Guilin, \\ Guangxi 541004, China - gzhou@glut.edu.cn \\ ${ }^{2}$ Department of Mechanical and Control Engineering, Guilin University of Technology, No. 12 Jian'gan Road, Guilin, Guangxi \\ 541004, China - (102016477, zqx0711)@glut.edu.cn \\ ${ }^{3}$ College of Science, Guilin University of Technology, Guilin 541004, PR China
}

KEY WORDS: Image classification, Quantum entanglement, Multispectral remote sensing

\begin{abstract}
:
Aiming at the problems of low accuracy and slow speed in the current remote sensing image classification algorithm,In order to improve remote sensing image classification, a quantum entanglement algorithm is proposed.The model transforms the classification process of remote sensing image into a random self-organization process of quantum particles in the state configuration space. The state configuration formed by entanglement of quantum particles evolves with time and finally converges to an average probability distribution.Taking Kunming city of Yunnan province as the research area, this paper compares the classification method in this paper with the traditional remote sensing classification method by using the $02 \mathrm{C}$ image data of yuanyuan1.Compared with other classification methods, the classification accuracy of this paper meets the requirements.
\end{abstract}

\section{INTRODUCTION}

Remote sensing image classification is a hot topic of remote sensing information extraction.In fact, the classification of remote sensing images is to divide each pixel or region of an image into different categories according to certain rules according to its spectral features, spatial structure features or other information in different spectral segments.

Although people have done a lot of research work on image classification, there is no general image classification theory, so the proposed segmentation algorithms are mostly aimed at specific problems, and there is no general classification algorithm for all images.Among them, the quantum derivative algorithm based on the basic theoretical framework of quantum mechanics has achieved encouraging results in traditional computer image processing.In 2001, Eldar from the Massachusetts institute of technology proposed the quantum signal processing theory (QSP) and proposed the basic framework of QSP.Quantum genetic algorithm, quantum particle swarm optimization algorithm, quantum immune algorithm and other algorithms based on quantum optimization have been widely used and achieved some results.In this paper, an algorithm of remote sensing image classification based on generalized quantum particle model is proposed to improve the effect of remote sensing classification. This model transforms the classification process of remote sensing image into quantum entanglement self-organization process and realizes the classification of remote sensing image.

\section{THEROY AND ALGORITHM}

Generalized quantum particle model was proposed by Shuai et al in 2006. The model was improved on the basis of generalized cellular automata and generalized particle model.Generalized cellular automaton model of cellular neural networks, cellular automata and ant colony algorithm, the traditional cellular automata is proposed on the basis of state of the nucleus, cell, harmonic functions such as concept, make cell evolution rules and generalized cellular automata parallel algorithm, make cell array on each cell according to the rules of evolution to reach the stationary probability distribution.Generalized particle model is developed on the basis of generalized cellular automata. The main difference between generalized cellular automata and generalized cellular automata is that every particle in the array of generalized particle model can move freely, while every cell in generalized cellular automata has a fixed position, but its cell state has changed.On the basis of generalized cellular automata, the generalized particle model improves the particle harmonic function, transfer frequency and evolution rules, and further improves the model.The generalized quantum particle model introduces the characteristics of quantum entanglement into the model, and the self-organizing clustering of this model is quite different from the previous similar applications of generalized cellular automata and generalized particle model.All of these particles can be randomly distributed on the array.Moreover, particles in each entangled class carry similar data objects, and the resulting entanglement distribution can be obtained by quantum measurement.

In this paper, a grayscale remote sensing image is transformed into a generalized quantum particle array in which each quantum particle represents a pixel.In this paper, a quantum entanglement theory for remote sensing image classification is proposed to simulate the evolution of quantum particles.Quantum state can be divided into pure state and mixed state. When expressed by density matrix, one quantum state is denoted as

* Corresponding author: Jianrong Xiao; E-mail: xjr@glut.edu.cn 


$$
\rho=\sum_{\mathrm{i}} P_{i}\left|\varphi_{i}><\varphi_{i}\right|
$$

Entangled state is a kind of quantum state. The entangled state means that the quantum state of the complex system under any representation cannot be written as the direct product of the wave functions of each subsystem.A classical bit can only be in one of two different states ( 0 and 1$)$, while the state of a quantum bit can be the superposition state of two different states, which can generally be described by a two-dimensional complex vector:

$$
|\phi>=\alpha| 0>+\beta \mid 1>
$$

Where the right vectors $\mid 0>$ and $\mid 1>$ are a set of orthogonal basis vectors to each other in the Hilbert space. $\alpha$ and $\beta$ are the quantum state $\phi$ with the probability amplitude of collapse, $\phi$ becoming 0 or 1 , so $|\alpha| 2+|\beta| 2=1$.

The state of two quantum bits $|\phi\rangle$ is described by the $H \otimes H$ vector on the tensor space, which can be generally expressed as

$$
>=\alpha_{11}\left|00>+\alpha_{12}\right| 01>+\alpha_{21}\left|10>+\alpha_{22}\right| 11>
$$

At this point, $\mid \varphi>$ can be divided into two different state vectors, one is the direct product state:

$$
\left(\alpha_{1}\left|0>+\beta_{1}\right| 1>\right) \otimes\left(\alpha_{2}\left|0>+\beta_{2}\right| 1>\right)
$$

The other kind of state is entangled state, which can not be defined as direct product form is called separable state, which means there is no quantum entanglement between two quantum bits in the state.The physical measurement of the first quantum bit does not affect the measurement of the second bit. The other type of state is the entangled state, and the definition of $|\varphi\rangle$ which cannot be written as a direct product is the entangled state.That's the entangled state.namely:

$$
\begin{gathered}
|\varphi>\neq| \varphi_{1}>\otimes \mid \varphi_{2}> \\
\frac{1}{\sqrt{2}}(|00>+| 11>)
\end{gathered}
$$

For example, formula (6) gives the EPR pair of two quantum bits, and the state of the system is entangled.The measurement of the first quantum bit directly changes the state of the second bit.In physics, two particles are said to be entangled if the measurement of one affects the measurement of the other.

In the process of remote sensing gray image classification, the remote sensing gray image is firstly transformed into a generalized quantum particle model array, in which the particles in the generalized quantum particle model array collide with the particles in their surrounding areas.If quantum entanglement theory is used to determine whether there is quantum entanglement between particles, if there is quantum entanglement between particles, the particles will self-organize into a class of entanglement.A remote sensing gray image is classified into quantum entangled particle arrays with multiple entanglement classes.

\section{A. Rules of the collision}

The neighborhood of coordinate point $(\mathrm{x}, \mathrm{y})$ in the generalized quantum particle model is defined as follows:

$$
N G(x, y)=\{(x-1, y),(x+1, y),(x, y-1),(x, y+1)\}
$$

Each dot in figure 1 represents a coordinate point of the quantum particle model. If and only if a quantum particle is in the neighborhood of another quantum particle, a collision occurs between the two particles.If two particles collide with each other and carry similar data objects, they become entangled, forming entangled quantum states.
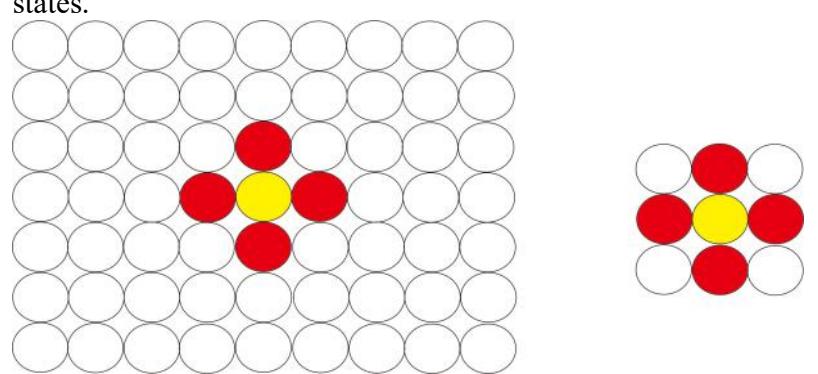

Figure 1. Coordinate neighborhood definition

\section{B. Entanglement rules}

Entanglement between quantum particles is the key to selforganizing clustering of quantum particles.Suppose any two quantum particles meet the condition of quantum entanglement, and they realize entanglement based on the following rules:

Two quantum particles is $\mathrm{P}_{1}$ and $\mathrm{P}_{2}$ respectively, its quantum state for:

$\left|\mathrm{P}_{1}>=\alpha_{1}\right| 0>+\beta_{1}|| 1>$ and $\left|\mathrm{P}_{2}>=\alpha_{2}\right| 0>+\beta_{2}|| 1>$.

The quantum entanglement state of $\mathrm{P}_{1}$ and $\mathrm{P}_{2}$ is $\left|\mathrm{P}_{1} \mathrm{P}_{2}>=\alpha\right| 0>+\beta \mid 1>$, where

$$
\left\{\begin{array}{l}
\alpha=\sqrt{\frac{\alpha_{1} / \alpha_{2}}{\alpha_{1} / \alpha_{2}+\beta_{1} / \beta_{2}}} \\
\beta=\sqrt{\frac{\beta_{1} / \beta_{2}}{\alpha_{1} / \alpha_{2}+\beta_{1} / \beta_{2}}}
\end{array}\right\}
$$

Formula (8) meets the following requirements:

1. The closer the probability amplitude corresponding to the quantum state of two quantum particles is, the closer the probability amplitude of the new entangled quantum state will be $1 / \sqrt{2}$.

2. The further the corresponding probability amplitude of the quantum state of two quantum particles is, the further the probability amplitude of the new entangled quantum state is $1 / \sqrt{2}$.

3. When the corresponding probability amplitude of the quantum state of two particles is equal, the probability amplitude of the entangled new quantum state is equal to $1 / \sqrt{2}$.

\section{Iteration rules}

When more than two quantum particles need to be entangled, the entanglement rule is improved as follows: suppose quantum particles $\mathrm{a}$ and $\mathrm{b}$ are entangled, and quantum particles $\mathrm{c}$ are also in the coordinate neighborhood of $\mathrm{a}$, and they meet the condition of quantum entanglement. According to the transitivity of the entanglement state, a,b and $\mathrm{c}$ will be entangled together. When a new quantum particle is entangled with any of a, b, or c, they form a class of entanglement.Each quantum particle is iterated repeatedly until the classification is completed. 


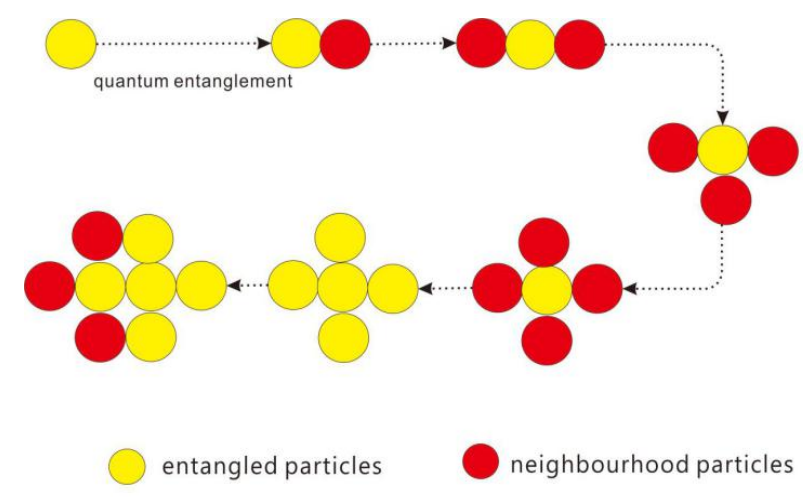

Figure 2. Process of Quantum entanglement

\section{TEXPERIMENT AND ANALYSIS}

The test area and data set of this paper: resource $102 \mathrm{C}$ satellite, the spectrum range is $(0.51-0.85 \mu \mathrm{m}, 0.52-0.59 \mu \mathrm{m}$, $0.63-0.69 \mu \mathrm{m}, \quad 0.77-0.89 \mu \mathrm{m})$.Multi-spectral remote sensing image at the junction of xishan district, puning county, kunming city, yunnan province $(240 \mathrm{~N}, 1020 \mathrm{E})$.Compared with Isodata method and k-means method, the results are as follows:

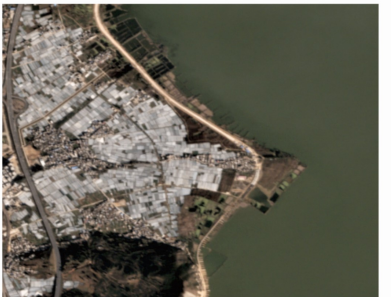

(a)

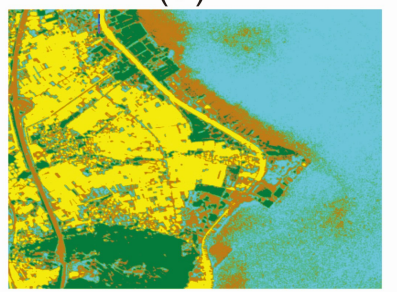

(c)

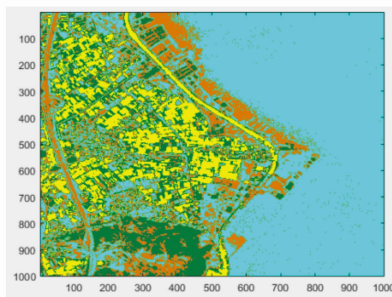

(b)

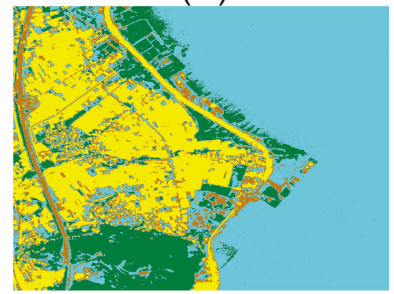

(d)

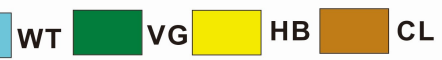

Figure 3. The results of classification in the third test area by: (a)the original image; (b) the proposed method; (c) Isodata; (d) K-Means

Table 1. Confusion matrix of the proposed method

\begin{tabular}{ccccccc}
\hline & WT & VG & HB & CL & PA & UA \\
\hline WT & 849 & 29 & 9 & 77 & 0.846 & 0.852 \\
VG & 37 & 963 & 28 & 58 & 0.917 & 0.861 \\
HB & 4 & 0 & 951 & 16 & 0.951 & 1.00 \\
CL & 5 & 12 & 18 & 958 & 0.943 & 0.952 \\
AA & & & & & 0.914 & 0.916 \\
\hline
\end{tabular}

\section{CONCLUSION}

At present, the traditional classification method is still widely used in remote sensing applications.Because quantum entanglement has the characteristics of clustering, A quantum entanglement based remote sensing image classification algorithm is proposed.Although compared with other classification methods, this method meets the requirements of remote sensing image classification, but there are some shortcomings. Combining quantum entanglement theory with other methods is the future development trend.

\section{ACKNOWLEDGEMENTS}

This paper is financially supported by the National Natural Science of China under Grant numbers 41431179,41961065; G uangxi Innovative Development Grand Grant under the grant nu mber: GuikeAA18118038, GuikeAA18242048; the National Ke y Research and Development Program of China under Grant nu mbers 2016YFB0502501 and the BaGuiScholars program of $G$ uangxi (Guoqing Zhou)

\section{REFERENCES}

Cireșan D, Meier U, Schmidhuber J. Multi-column deep neural networks for image classification[J]. arXiv preprint arXiv:1202.2745, 2012.

Chapelle O, Haffner P, Vapnik V N. Support vector machines for histogram-based image classification[J]. IEEE transactions on Neural Networks, 1999, 10(5): 1055-1064.

Hinterding R. Representation, Constraint Satisfaction and the Knapsack Problem[C]. The IEEE Congress on Evolutionary Computation,Washington D C,USA 1999

J. D. Farmer, N. H. Packard, A. S. Perelson. The immune system, adaptation, and machine learning[J]. Physica D: Nonlinear Phenomena, 1986, 22(1): 187-204

Latorre J I. Image compression and entanglement[J]. arXiv preprint quant-ph/0510031, 2005.

Shuai, D., Liu, Y., Shuai, Q., Huang, L., \& Dong, Y. (2006). Self-Organizing Data Clustering Based on Quantum Entanglement Model. First International Multi-Symposiums on Computer and Computational Sciences (IMSCCS'06).

Sun J, Feng B, Xu W. Particle Swarm Optimization with Particles Having Quantum Behavior[C]. IEEE Congress on Evolutionary Computation, California, USA, 2004

Wang J, Yang J, Yu K, et al. Locality-constrained linear coding for image classification[C]//2010 IEEE computer society conference on computer vision and pattern recognition. IEEE, 2010: 3360-3367.

Zhang, G., Zhang, R., Zhou, G., \& Jia, X.Hierarchical spatial features learning with deep CNNs for very high-resolution remote sensing image classification. International Journal of Remote Sensing, 2018,1-19. 
The International Archives of the Photogrammetry, Remote Sensing and Spatial Information Sciences, Volume XLII-3/W10, 2020 International Conference on Geomatics in the Big Data Era (ICGBD), 15-17 November 2019, Guilin, Guangxi, China

Zhou, G., Zhang, R., \& Zhang, D. Manifold learning colocation decision tree for remotely sensed imagery classification. Remote Sensing, 2016,8(10), 855. 